\title{
Generating Question Relevant Captions to Aid Visual Question Answering
}

\author{
Jialin Wu, Zeyuan Hu and Raymond J. Mooney \\ Department of Computer Science \\ University of Texas at Austin \\ \{jialinwu, iamzeyuanhu, mooney\}ecs.utexas.edu
}

\begin{abstract}
Visual question answering (VQA) and image captioning require a shared body of general knowledge connecting language and vision. We present a novel approach to improve VQA performance that exploits this connection by jointly generating captions that are targeted to help answer a specific visual question. The model is trained using an existing caption dataset by automatically determining question-relevant captions using an online gradient-based method. Experimental results on the VQA v2 challenge demonstrates that our approach obtains state-of-the-art VQA performance (e.g. $68.4 \%$ on the Test-standard set using a single model) by simultaneously generating question-relevant captions.
\end{abstract}

\section{Introduction}

In recent years, visual question answering (VQA) (Antol et al., 2015) and image captioning (Donahue et al., 2015; Rennie et al., 2017) have been widely studied in both the computer vision and NLP communities. Most recent VQA research (Lu et al., 2017; Pedersoli et al., 2017; Anderson et al., 2018; Lu et al., 2018) concentrates on directly utilizing visual input features including detected objects, attributes, and relations between pairs of objects.

However,little VQA research works on exploiting textual features from the image which are able to tersely encode the necessary information to answer the questions. This information could be richer than the visual features in that the sentences have fewer structural constraints and can easily include the attributes of and relation among multiple objects. In fact, we observe that appropriate captions can be very useful for many VQA questions. In particular, we trained a model to answer visual questions for the VQA v2 challenge (Antol et al., 2015) only using the human annotated

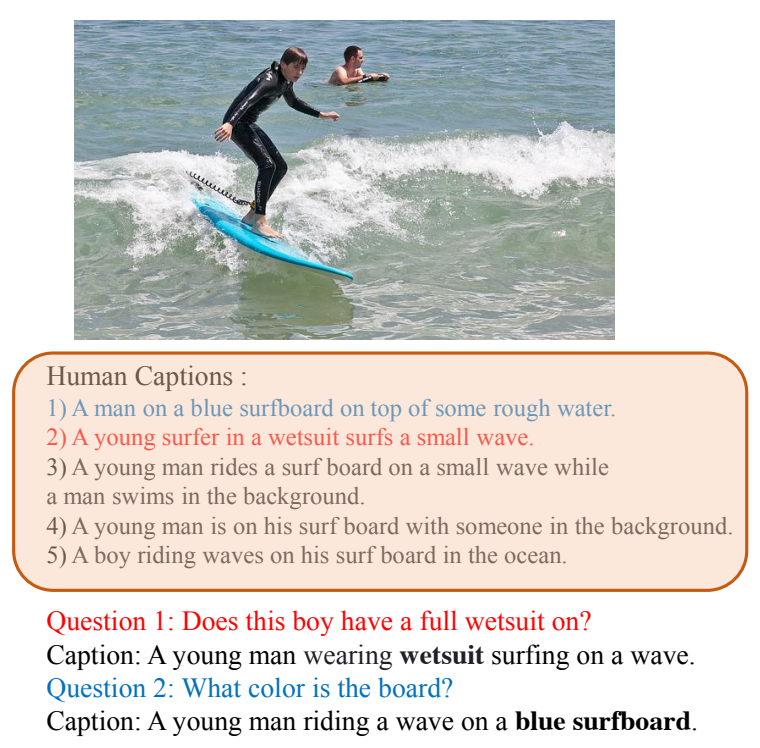

Figure 1: Examples of our generated question-relevant captions. During the training phase, our model selects the most relevant human captions for each question (marked by the same color).

captions without images and achieved a score of $59.6 \%$, outperforming a large number of VQA models that use image features. Existing work using captions for VQA has generated questionagnostic captions using a pretrained captioner ( $\mathrm{Li}$ et al., 2018a). This approach can provide additional general information; however, this information is not guaranteed to be relevant to the given VQA question.

We explore a novel approach that generates question-relevant image descriptions, which contain information that is directly relevant to a particular VQA question. Fig. 1 shows examples of our generated captions given different questions. In order to encourage the generation of relevant captions, we propose a novel greedy algorithm that aims to minimize the cross entropy loss only for 
the most relevant and helpful gold-standard captions. Specifically, helpfulness is measured using the inner-product of the gradients from the caption generation loss and the VQA answer prediction loss. A positive inner-product means the two objective functions share some descent directions in the optimization process, and therefore indicates that the corresponding captions help the VQA training process.

In order to incorporate the caption information, we propose a novel caption embedding module that, given the question and image features for a visual question, recognizes important words in the caption, and produces a caption embedding tailored for answer prediction. In addition, the caption embeddings are also utilized to adjust the visual top-down attention weights for each object.

Furthermore, generating question-relevant captions ensures that both image and question information is encoded in their joint representations, which reduces the risk of learning from question bias (Li et al., 2018a) and ignoring the image content when high accuracy can be achieved from the questions alone.

Experimental evaluation of our approach shows significant improvements on VQA accuracy over our baseline Up-Down (Anderson et al., 2018) model on the VQA v2 validation set (Antol et al., 2015), from $63.2 \%$ to $67.1 \%$ with gold-standard human captions from the COCO dataset (Chen et al., 2015) and $65.8 \%$ with automatically generated question-relevant captions. Our single model is able to score $68.4 \%$ on the test-standard split, and an ensemble of 10 models scores $69.7 \%$.

\section{Background Related Work}

\subsection{Visual Question Answering}

Recently, a large amount of attention-based deeplearning methods have been proposed for VQA, including top-down (Ren et al., 2015a; Fukui et al., 2016; Wu et al., 2016; Goyal et al., 2017; Li et al., 2018a) and bottom-up attention methods (Anderson et al., 2018; Li et al., 2018b; Wu and Mooney, 2019). Specifically, a typical model first extracts image features using a pre-trained $\mathrm{CNN}$, and then trains an RNN to encode the question, using an attention mechanism to focus on specific features of the image. Finally, both question and attended image features are used to predict the final answer.

However, answering visual questions requires not only information about the visual content but also common knowledge, which is usually too hard to directly learn from only a limited number of images with human annotated answers as supervision. However, comparatively little previous VQA research has worked on enriching the knowledge base. We are aware of two related papers. Li et al. (2018a) use a pre-trained captioner to generate general captions and attributes with a fixed annotator and then use them to predict answers. Therefore, the captions they generate are not necessarily relevant to the question, and they may ignore image features needed for answer prediction. Narasimhan et al. (2018) employed an out-of-thebox knowledge base and trained their model to filter out irrelevant facts. After that, graph convolutional networks use this knowledge to build connections to the relevant facts and predict the final answer. Unlike them, we generate captions to provide information that is directly relevant to the VQA process.

\subsection{Image Captioning}

Most recent image captioning models are also attention-based deep-learning models (Donahue et al., 2015; Karpathy and Fei-Fei, 2015; Vinyals et al., 2015; Luo et al., 2018; Liu et al., 2018). With the help of large image description datasets (Chen et al., 2015), these models have demonstrated remarkable results. Most of them encode the image using a CNN, and build an attentional RNN (i.e. GRU (Cho et al., 2014), LSTM (Hochreiter and Schmidhuber, 1997)) on top of the image features as a language model to generate image captions.

However, deep neural models still tend to generate general captions based on the most significant objects (Vijayakumar et al., 2016). Although previous works (Luo et al., 2018; Liu et al., 2018) build captioning models that are encouraged to generate different captions with discriminability objectives, the captions are usually less informative and fail to describe most of the objects and their relationships diversely. In this work, we develop an approach to generating captions that directly focus on the critical objects in the VQA process and provide information that can help the VQA module predict the answer.

\section{Approach}

We first describe the overall structure of our joint model in Sec. 3.1 and explain the foundational 


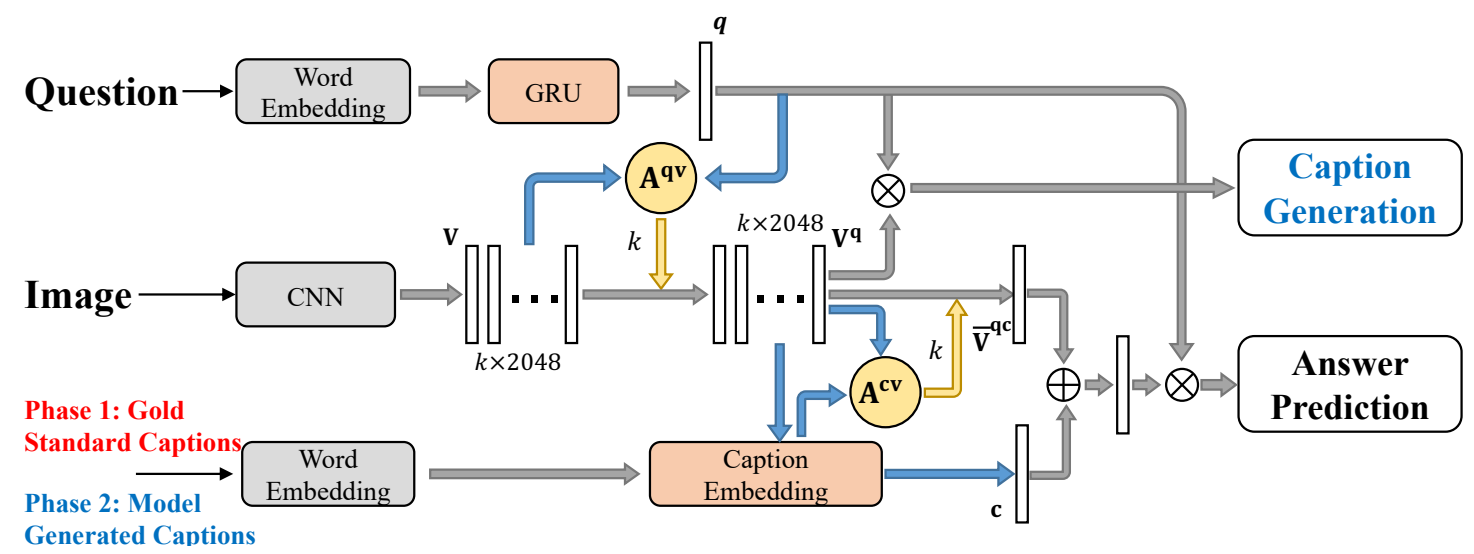

Figure 2: Overall structure of our model that generates question-relevant captions to aid VQA. Our model is first trained to generate question-relevant captions as determined in an online fashion in phase 1 . Then, the VQA model is fine-tuned with generated captions from the first phase to predict answers. $\otimes$ denotes element-wise multiplication and $\oplus$ denotes element-wise addition. Blue arrows denote fully-connected layers $(f c)$ and yellow arrows denote attention embedding.

feature representations (i.e. image, question and caption embeddings) in Sec. 3.2. Then, the VQA module is presented in Sec. 3.3, which takes advantage of the generated image captions to improve the VQA performance. In Sec. 3.4, we explain the image captioning module which generates question-relevant captions. Finally, the training and implementation details are provided in Sec. 3.5.

\subsection{Overview}

As illustrated in Fig. 2, the proposed model first extracts image features $\mathbf{V}=\left\{\mathbf{v}_{1}, \mathbf{v}_{2}, \ldots, \mathbf{v}_{K}\right\}$ using bottom-up attention and question features $\mathbf{q}$ to produce their joint representation and then generates question-related captions. Next, our caption embedding module encodes the generated captions as caption features $\mathbf{c}$ as detailed in Sec. 3.2. After that, both question features $\mathbf{q}$ and caption features $\mathbf{c}$ are utilized to generate the visual attention $\mathbf{A}^{c v}$ to weight the images' feature set $\mathbf{V}$, producing attended image features $\overline{\mathbf{v}}^{q c}$. Finally, we add $\overline{\mathbf{v}}^{q c}$ to the caption features $\mathbf{c}$ and further perform element-wise multiplication with the question features $\mathbf{q}$ (Anderson et al., 2018) to produce the joint representation of the question, image and caption, which is then used to predict the answer.

\subsection{Feature Representation}

In this section, we explain the details of this joint representation. We use $f(x)$ to denote fullyconnected layers, where $f(x)=\operatorname{LReLU}(W x+b)$ with input features $x$ and ignore the notation of weights and biases for simplicity, where these $f c$ layers do not share weights. LReLU denotes a Leaky ReLU (He et al., 2015).

\section{Image and Question Embedding}

We use object detection as bottom-up attention (Anderson et al., 2018), which provides salient image regions with clear boundaries. In particular, we use a Faster R-CNN head (Ren et al., 2015b) in conjunction with a ResNet-101 base network (He et al., 2016) as our detection module. The detection head is first pre-trained on the Visual Genome dataset (Krishna et al., 2017) and is capable of detecting 1,600 objects categories and 400 attributes. To generate an output set of image features $\mathbf{V}$, we take the final detection outputs and perform non-maximum suppression (NMS) for each object category using an IoU threshold of 0.7 . Finally, a fixed number of 36 detected objects for each image are extracted as the image features (a 2, 048 dimensional vector for each object) as suggested by Teney et al. (2017).

For the question embedding, we use a standard GRU (Cho et al., 2014) with 1,280 hidden units and extract the output of the hidden units at the final time step as the question features $\mathbf{q}$. Following Anderson et al. (2018), the question features $\mathbf{q}$ and image feature set $\mathbf{V}$ are further embedded together to produce a question-attended image feature set $\mathbf{V}^{q}$ via question visual-attention $\mathbf{A}^{q v}$ as illustrated in Fig. 2.

\section{Caption Embedding}

Our novel caption embedding module takes as in- 
put the question-attended image feature set $\mathbf{V}^{q}$, question features $\mathbf{q}$, and $C$ captions $\mathbf{W}_{i}^{c}=$ $\left\{w_{i, 1}^{c}, w_{i, 2}^{c}, \ldots, w_{i, T}^{c}\right\}$, where $T$ denotes the length of the captions and $i=1, \ldots, C$ are the caption indices, and then produces the caption features $\mathbf{c}$.

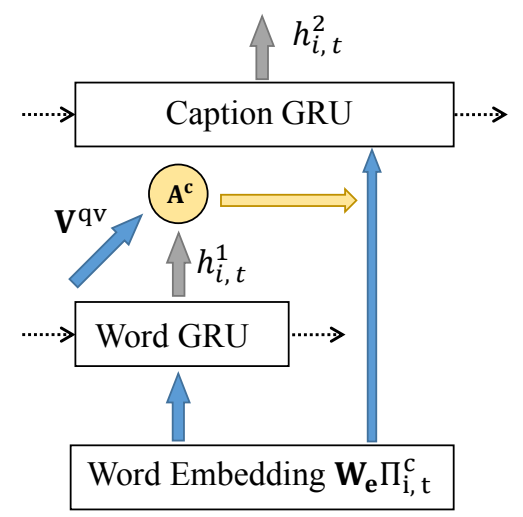

Figure 3: Overview of the caption embedding module. The Word GRU is used to generate attention to identify the relevant words in each caption, and the Caption GRU generates the final caption embedding. We use question-attended image features $\mathbf{V}^{q v}$ to compute the attention. Blue arrows denote $f c$ layers and yellow arrows denote attention embedding.

The goals of the caption module are to serve as a knowledge supplement to aid VQA, and to provide additional clues to identify the relevant objects better and adjust the top-down attention weights. To achieve this, as illustrated in Fig. 3, we use a two-layer GRU architecture. The firstlayer GRU (called the Word GRU) sequentially encodes the words in a caption $\mathbf{W}_{i}^{c}$ at each time step as $h_{i, t}^{1}$.

$$
h_{i, t}^{1}=\operatorname{GRU}\left(\mathbf{W}_{e} \Pi_{i, t}^{c}, h_{i, t-1}^{1}\right)
$$

where $\mathbf{W}_{e}$ is the word embedding matrix, and $\Pi_{i, t}^{c}$ is the one-hot embedding for the word $w_{i, t}^{c}$.

Then, we design a caption attention module $\mathbf{A}^{c}$ which utilizes the question-attended feature set $\mathbf{V}^{q}$, question features $\mathbf{q}$, and $h_{i, t}^{1}$ to generate the attention weight on the current word in order to indicate its importance. Specifically, the Word GRU first encodes the words embedding $\Pi_{i, t}^{c}$ in Eq. 1, and then we feed the outputs $h_{i, t}^{1}$ and $\mathbf{V}^{q}$ to the attention module $\mathbf{A}^{c}$ as shown in Eq. 4.

$$
\begin{aligned}
\overline{\mathbf{v}}^{q} & =\sum_{k=1}^{K} \mathbf{v}_{k}^{q} \\
a_{i, t}^{c} & =h_{i, t}^{1} \circ f\left(\overline{\mathbf{v}}^{q}\right)+h_{i, t}^{1} \circ f(\mathbf{q}) \\
\alpha_{i, t}^{c} & =\sigma\left(a_{i, t}^{c}\right)
\end{aligned}
$$

where $\sigma$ denotes the sigmoid function, and $K$ is the number of objects in the bottom-up attention.

Next, the attended words in the caption are used to produce the final caption representation in Eq. 5 via the Caption GRU. Since the goal is to gather more information, we perform element-wise max pooling across the representations of all of the input captions $\mathbf{c}_{i}$ in Eq. 7.

$$
\begin{aligned}
h_{i, t}^{2} & =\operatorname{GRU}\left(\alpha_{i, t}^{c} \mathbf{W}_{e} \Pi_{i, t}^{c}, h_{i, t-1}^{2}\right) \\
\mathbf{c}_{i} & =f\left(h_{i, T}^{2}\right) \\
\mathbf{c} & =\max \left(\mathbf{c}_{i}\right)
\end{aligned}
$$

where $\max$ denotes the element-wise max pooling across all of caption representations $\mathbf{c}_{i}$ of the image.

\subsection{VQA Module}

This section describes the details of the VQA module. The generated captions are usually capable of capturing relations among the questionrelevant objects; however these relations are absent in the bottom-up attention. Therefore, our VQA module utilizes the caption embeddings $\mathbf{c}$ to adjust the top-down attention weights in VQA in order to produce the final caption-attended features $\overline{\mathbf{v}}^{q c}$ in Eq. 10:

$$
\begin{aligned}
a_{k}^{c v} & =f\left(f(\mathbf{c}) \circ f\left(\overline{\mathbf{v}}_{k}^{q}\right)\right) \\
\alpha_{k}^{c v} & =\operatorname{softmax}\left(a_{c, k}^{c v}\right) \\
\overline{\mathbf{v}}^{q c} & =\sum_{k}^{K} \mathbf{v}_{k}^{q} \alpha_{k}^{c v}
\end{aligned}
$$

where $k$ traverses the $K$ objects features.

To better incorporate the information from the captions into the VQA process, we add the caption features $\mathbf{c}$ to the attended image features $\overline{\mathbf{v}}^{q c}$, and then element-wise multiply by the question features as shown in Eq. 11:

$$
\begin{aligned}
\mathbf{h} & =\mathbf{q} \circ\left(f\left(\overline{\mathbf{v}}^{q c}\right)+f(\mathbf{c})\right) \\
\hat{s} & =\sigma(f(\mathbf{h}))
\end{aligned}
$$

We frame the answer prediction task as a multilabel regression problem (Anderson et al., 2018). In particular, we use the soft scores in the goldstandard VQA-v2 data (which are used in the evaluation metric), as labels to supervise the sigmoidnormalized predictions as shown in Eq. 13:

$$
\mathcal{L}^{v q a}=-\sum_{j=1}^{N} s_{j} \log \hat{s}_{j}+\left(1-s_{j}\right) \log \left(1-\hat{s}_{j}\right)
$$


where the index $j$ runs over $N$ candidate answers and $s$ are the soft answer scores.

In case of multiple feasible answers, the soft scores capture the occasional uncertainty in the ground-truth annotations. As suggested by Teney et al. (2017), we collect the candidate answers that appear more than 8 times in the training set, which results in 3,129 answer candidates.

\subsection{Image Captioning Module}

We adopt an image captioning module similar to that of Anderson et al. (2018), which takes the object detection features as inputs and learns attention weights over those objects' features in order to predict the next word at each step. The key difference between our module and theirs lies in the input features and the caption supervision. Specifically, we use the question-attended image features $\mathbf{V}^{q}$ as inputs, and only use the most relevant caption, which is automatically determined in an online fashion (detailed below), for each question-image pair to train the captioning module. This ensures that only question-relevant captions are generated.

\section{Selecting Relevant Captions for Training}

Previously, Li et al. (2018b) selected relevant captions for VQA based on word similarities between captions and questions, however, their approach does not take into account the details of the VQA process. In contrast, during training, our approach dynamically determines for each problem, the caption that will most improve VQA. We do this by updating with a shared descent direction (Wu et al., 2018) which decreases the loss for both captioning and VQA. This ensures a consistent target for both the image captioning module and the VQA module in the optimization process.

During training, we compute the cross-entropy loss for the $i$-th caption using Eq. 14, and backpropagate the gradients only from the most relevant caption determined by solving Eq. 15 .

$$
\mathcal{L}_{i}^{c}=-\sum_{t=1}^{T} \log \left(p\left(w_{i, t}^{c} \mid w_{i, t-1}^{c}\right)\right)
$$

In particular, we require the inner product of the current gradient vectors from the predicted answer and the human captions to be greater than a positive constant $\xi$, and further select the caption that maximizes that inner product.

$$
\begin{gathered}
\underset{i}{\arg \max } \sum_{k=0}^{K}\left(\frac{\partial \hat{s}_{\text {pred }}}{\partial \mathbf{v}_{k}^{q}}\right)^{T} \frac{\partial \log \left(p\left(\mathbf{W}_{i}^{c}\right)\right)}{\partial \mathbf{v}_{k}^{q}} \\
\text { s.t. } \sum_{k=0}^{K}\left(\frac{\partial \hat{s}_{\text {pred }}}{\partial \mathbf{v}_{k}^{q}}\right)^{T} \frac{\partial \log \left(p\left(\mathbf{W}_{i}^{c}\right)\right)}{\partial \mathbf{v}_{k}^{q}}>\xi
\end{gathered}
$$

where the $\hat{s}_{\text {pred }}$ is the logit ${ }^{1}$ for the predicted answer, $\mathbf{W}_{i}^{c}$ denotes the $i$-th human caption for the image and $k$ traverses the $K$ object features.

Therefore, given the solution to Eq. $15, i^{\star}$, the final loss of our joint model is the sum of the VQA loss and the captioning loss for the selected captions as shown in Eq. 16. If Eq. 15 has no feasible solution, we ignore the caption loss.

$$
\mathcal{L}=\mathcal{L}^{v q a}+\mathcal{L}_{i^{\star}}^{c}
$$

\subsection{Training and Implementation Details}

We train our joint model using the AdaMax optimizer (Kingma and Ba, 2015) with a batch size of 384 and a learning rate of 0.002 as suggested by Teney et al. (2017). We use the validation set for VQA v2 to tune the initial learning rate and the number of epochs, yielding the highest overall VQA score. We use 1,280 hidden units in the question embedding and attention model in the VQA module with 36 object detection features for each image. For captioning models, the dimension of the LSTM hidden state, image feature embedding, and word embedding are all set to 512. We also use Glove vectors (Pennington et al., 2014) to initialize the word embedding matrix in the caption embedding module.

We initialize the training process with human annotated captions from the $\mathrm{COCO}$ dataset (Chen et al., 2015) and pre-train the VQA and captiongeneration modules for 20 epochs with the final joint loss in Eq. 16. After that, we generate question-relevant captions for all question-image pairs in the COCO train, validation, and test sets. In particular, we sample 5 captions per questionimage pair. We fine-tune our model using the generated captions with $0.25 \times$ learning-rate for another 10 epochs.

\section{Experiments}

We perform extensive experiments and ablation studies to evaluate our joint model on VQA.

\footnotetext{
${ }^{1}$ The input to the softmax function.
} 


\begin{tabular}{l|ccc|c}
\hline & \multicolumn{4}{|c}{ Test-standard } \\
\hline & Yes/No & Num & Other & All \\
\hline \hline Prior (Goyal et al., 2017) & 61.20 & 0.36 & 1.17 & 25.98 \\
Language-only (Goyal et al., 2017) & 67.01 & 31.55 & 27.37 & 44.26 \\
MCB (Fukui et al., 2016) & 78.82 & 38.28 & 53.36 & 62.27 \\
Up-Down (Anderson et al., 2018) & 82.20 & 43.90 & 56.26 & 65.32 \\
VQA-E (Li et al., 2018b) & 83.22 & 43.58 & 56.79 & 66.31 \\
Ours(single) & $\mathbf{8 4 . 6 9}$ & $\mathbf{4 6 . 7 5}$ & $\mathbf{5 9 . 3 0}$ & $\mathbf{6 8 . 3 7}$ \\
\hline Ours(Ensemble-10) & $\mathbf{8 6 . 1 5}$ & $\mathbf{4 7 . 4 1}$ & $\mathbf{6 0 . 4 1}$ & $\mathbf{6 9 . 6 6}$ \\
\hline
\end{tabular}

Table 1: Comparison of our results on VQA with the state-of-the-art methods on the test-standard data. Accuracies in percentage $(\%)$ are reported.

\subsection{Datasets and Evaluation Metrics}

\section{VQA Dataset}

We use the VQA v2.0 dataset (Antol et al., 2015) for the evaluation of our proposed joint model, where the answers are balanced in order to minimize the effectiveness of learning dataset priors. This dataset is used in the VQA 2018 challenge and contains over $1.1 \mathrm{M}$ questions from the over $200 \mathrm{~K}$ images in the MSCOCO 2015 dataset (Chen et al., 2015).

Following Anderson et al. (2018), we perform standard text pre-processing and tokenization. In particular, questions are first converted to lower case and then trimmed to a maximum of 14 words, and the words that appear less than 5 times are replaced with an "<unk>" token. To evaluate answer quality, we report accuracies using the official VQA metric using soft scores, which accounts for the occasional disagreement between annotators for the ground truth answers.

\section{Image Captioning Dataset}

We use the MSCOCO 2014 dataset (Chen et al., 2015) for the image caption module. To maintain consistency with the VQA tasks, we use the dataset's official configuration that includes 82, 372 images for training and 40, 504 for validation. Similar to the VQA question pre-processing, we first convert all sentences to lower case, tokenizing on white spaces, and filtering words that do not occur at least 5 times.

\subsection{Results on VQA}

We first report the experimental results on the VQA task and compare our results with the stateof-the-art methods in this section. After that, we perform ablation studies to verify the contribution of additional knowledge from the generated captions, and the effectiveness of using caption representations to adjust the top-down visual attention weights.

As demonstrated in Table 1, our single model outperforms other state-of-the-art single models by a clear margin, i.e. $2.06 \%$, which indicates the effectiveness of including caption features as additional inputs. In particular, we observe that our single model outperforms other methods, especially in the 'Num' and 'Other' categories. This is because the generated captions are capable of providing more numerical clues for answering the 'Num' questions, since the captions can describe the number of relevant objects and provide general knowledge for answering the 'Other' questions. Furthermore, an ensemble of 10 models with different initialization seeds results in a score of $69.7 \%$ for the test-standard set.

Fig. 4 shows several examples of our generated question-relevant captions. These examples illustrate how different captions are generated for the same image when the question is changed. They also show how the objects in the image that are important to answering the question are described in the question-relevant captions.

\section{Comparison Between Using Generated and Human Captions}

Next, we analyze the difference between using automatically generated captions and using those provided by human annotators. In particular, we train our model with generated question-agnostic captions using the Up-Down (Anderson et al., 2018) captioner, question-relevant captions from our caption generation module, and human annotated captions from the $\mathrm{COCO}$ dataset.

As demonstrated in Table 2, our model gains 


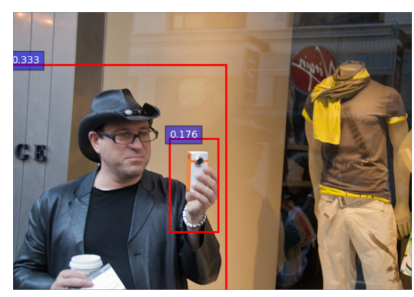

Q: What is he doing?

Caption: A man is taking a picture of himself with a phone.

A: Taking picture.

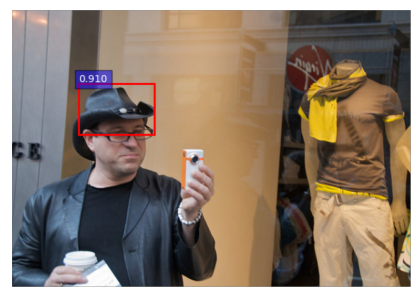

Q: Is he wearing a hat?

Caption: A man with glasses and $₫$ hat $\mathbb{0}$.

A: Yes.

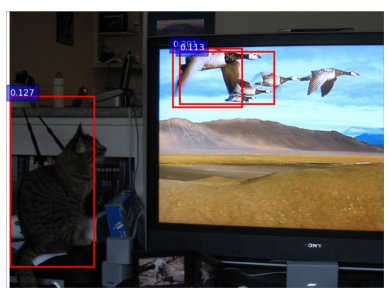

Q: Is the cat watching TV?

Caption: $\mathbb{A}$ cat is watching @ birred on the screen.

A: Yes.

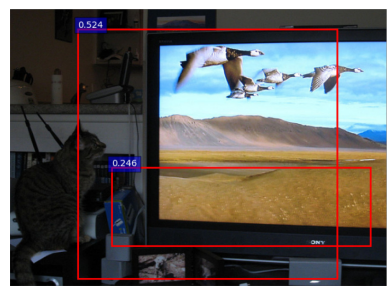

Q: Is the tv on?

Caption: A bird fllying om al lange television screen.

A: Yes.

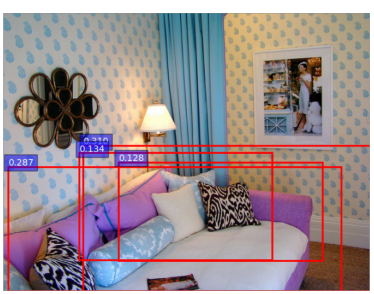

Q: What colors are on the couch? Caption: $\mathbb{A}$ living room with a blue and white bed.

A: Purple and white.

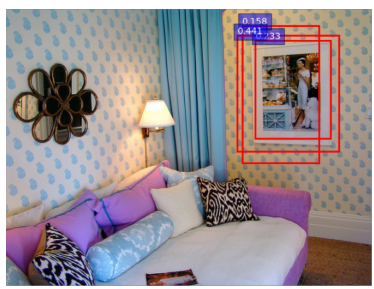

Q: Is there a picture on the wall?

Caption: A bediroom with pictures on the wall.

A: Yes.

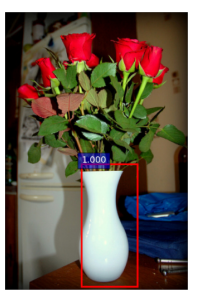

Q: What color is the vase? Caption: A white vase finlled. with lots of flowers. A: White.

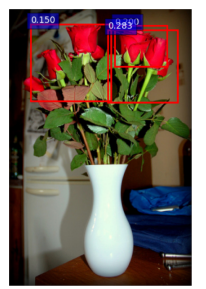

Q: What color are the flowers? lots of red roses. A: Red.

Figure 4: Examples of our generated question-relevant captions. The influential objects with attention weights greater than 0.1 are indicated by bounding boxes (annotated with their visual attention weights in the blue box), and the gray-scale levels in the caption words indicate the word attentions from the caption embedding module.

\begin{tabular}{l|c}
\hline & Validation \\
\hline Up-Down (Anderson et al., 2018) & 63.2 \\
Ours with Up-Down captions & 64.6 \\
Ours with our generated captions & 65.8 \\
Ours with human captions & $\mathbf{6 7 . 1}$ \\
\hline
\end{tabular}

Table 2: Comparison of the performance using generated and human captions. Both of them provide significant improvements to the baseline model. However, there is still a reasonable gap between generated and human captions.

about $4 \%$ improvement from using human captions and $2.5 \%$ improvement from our generated question-relevant captions on the validation set. This indicates the insufficiency of directly answering visual questions using a limited number of detection features, and the utility of incorporating additional information about the images. We also observe that our generated question-relevant captions trained with our caption selection strategy provide more helpful clues for the VQA process than the question-agnostic Up-Down captions, outperforming their captions by $1.2 \%$.

Effectiveness of Adjusting Top-Down Attention In this section, we quantitatively analyze the ef-

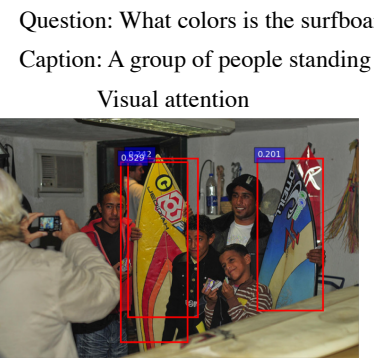

Answer: Yellow and blue
Answer: yellow and red to yellow board.

Caption adjusted visual attention

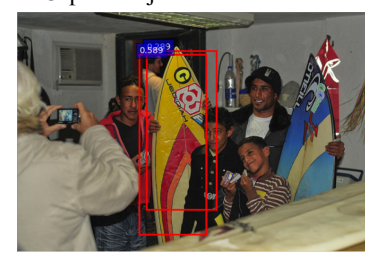

Answer: Yellow and red
Figure 5: An example of caption attention adjustment. The question-relevant caption helps the VQA module adjust the visual attention from both the yellow board and the blue sail to the yellow board only.

fectiveness of utilizing captions to adjust the topdown attention weights, in addition to the advantage of providing additional information. In particular, we compare our model with a baseline version where the top-down attention-weight adjustment factor $\mathbf{A}^{c v}$ is manually set to 1.0 (resulting in no adjustment).

As demonstrated in Tables 3 and 4, we observe an improvement when using caption features to adjust the attention weights. This indicates that the caption features help the model to more robustly locate the objects that are helpful to the VQA pro- 
cess. We use $w \mathcal{C A} \mathcal{A}$ to indicate with caption attention adjustment and $w / o \mathcal{C A} \mathcal{A}$ to indicate without it. Fig. 5 illustrates an example of caption attention adjustment. Without $\mathcal{C} \mathcal{A} \mathcal{A}$, the top-down visual attention focuses on both the yellow surfboard and the blue sail, generating the incorrect answer "yellow and blue.". However, with "yellow board" in the caption, the caption attention adjustment $(\mathcal{C} \mathcal{A} \mathcal{A})$ helps the VQA module focus attention just on the yellow surfboard, thereby generating the correct answer "yellow and red" (since there is some red coloring in the surfboard).

\begin{tabular}{l|cccc}
\hline & \multicolumn{4}{|c}{ Test-standard } \\
\hline & All & Yes/No & Num & Other \\
\hline \hline Up-Down & 65.3 & 82.2 & 43.9 & 56.3 \\
Ours $w / o \mathcal{C} \mathcal{A} \mathcal{A}$ & 67.4 & 84.0 & 44.5 & 57.9 \\
Ours $w \mathcal{C} \mathcal{A} \mathcal{A}$ & 68.4 & 84.7 & 46.8 & 59.3 \\
\hline
\end{tabular}

Table 3: Evaluation of the effectiveness of captionbased attention adjustment $(\mathcal{C} \mathcal{A} \mathcal{A})$ on the test-standard data. Accuracies in percentage (\%) are reported.

\begin{tabular}{l|cccc}
\hline & \multicolumn{4}{|c}{ Validation } \\
\hline & All & Yes/No & Num & Other \\
\hline \hline Up-Down & 63.2 & 80.3 & 42.8 & 55.8 \\
Ours $w / o \mathcal{C} \mathcal{A} \mathcal{A}$ & 65.2 & 82.1 & 43.6 & 55.8 \\
Ours $w \mathcal{C} \mathcal{A} \mathcal{A}$ & 65.8 & 82.6 & 43.9 & 56.4 \\
\hline
\end{tabular}

Table 4: Evaluation of the effectiveness of $\mathcal{C} \mathcal{A} \mathcal{A}$ on the validation data. Accuracies in percentage $(\%)$ are reported.

Next, in order to directly demonstrate that our generated question-relevant captions help the model to focus on more relevant objects via attention adjustment, we compare the differences between the generated visual attention and humanannotated important objects from the VQA-X dataset (Park et al., 2018), which has been used to train and evaluate multimodal (visual and textual) VQA explanation (Wu and Mooney, 2018). The VQA-X dataset contains 2,000 question-image pairs from the VQA v2 validation set with human annotations indicating the objects which most influence the answer to the question. In particular, we used Earth Mover Distance (EMD) (Rubner et al., 2000) to compare the highly-attended objects in the VQA process to the objects highlighted by human judges. This style of evaluation using EMD has previously been employed to compare automatic visual explanations to humanattention annotations (Selvaraju et al., 2017; Park et al., 2018).

We resize all of the 2,000 human annotations in VQA-X dataset to $14 \times 14$ and adjust the object bounding boxes in the images accordingly. Next, we assign the top-down attention weights to the corresponding bounding boxes, both before and after caption attention adjustment, and add up the weights of all 36 detections. Then, we normalize attention weights over the $14 \times 14$ resized images to sum to one, and finally compute the EMD between the normalized visual attentions and the human annotations.

Table 5 reports the EMD results for the attentions weights both before and after the caption attention adjustments.

\begin{tabular}{l|c|c|c}
\hline & $w / o \mathcal{C} \mathcal{A} \mathcal{A}$ & $w \mathcal{C} \mathcal{A} \mathcal{A}$ & Human \\
\hline EMD & 2.38 & 2.30 & 2.25 \\
\hline
\end{tabular}

Table 5: EMD results comparing the top-down attention weights (with or without caption attention adjustments) to human attention-annotation from the VQA$\mathrm{X}$ dataset. Results are shown for both automatically generated captions and human captions. Lower EMD indicates a closer match to human attention.

The results indicate that caption attention adjustment improves the match between automated attention and human-annotated attention, even though the approach is not trained on supervised data for human attention. Not surprisingly, human captions provide a bit more improvement than automatically generated ones.

\section{Conclusion}

In this work, we have explored how generating question-relevant image captions can improve VQA performance. In particular, we present a model which jointly generates question-related captions and uses them to provide additional information to aid VQA. This approach only utilizes existing image-caption datasets, automatically determining which captions are relevant to a given question. In particular, we design the training algorithm to only update the network parameters in the optimization process when the caption generation and VQA tasks agree on the direction of change. Our single model joint system outperforms the current state-of-the-art single model for VQA. 


\section{Acknowledgement}

This research was supported by the DARPA XAI program under a grant from AFRL.

\section{References}

Peter Anderson, Xiaodong He, Chris Buehler, Damien Teney, Mark Johnson, Stephen Gould, and Lei Zhang. 2018. Bottom-Up and Top-Down Attention for Image Captioning and VQA. In CVPR, volume 3 , page 6 .

Stanislaw Antol, Aishwarya Agrawal, Jiasen Lu, Margaret Mitchell, Dhruv Batra, C Lawrence Zitnick, and Devi Parikh. 2015. VQA: Visual Question Answering. In Proceedings of the IEEE International Conference on Computer Vision, pages 2425-2433.

Xinlei Chen, Hao Fang, Tsung-Yi Lin, Ramakrishna Vedantam, Saurabh Gupta, Piotr Dollár, and C Lawrence Zitnick. 2015. Microsoft COCO Captions: Data Collection and Evaluation Server. arXiv preprint arXiv:1504.00325.

Kyunghyun Cho, Bart Van Merriënboer, Caglar Gulcehre, Dzmitry Bahdanau, Fethi Bougares, Holger Schwenk, and Yoshua Bengio. 2014. Learning Phrase Representations Using RNN EncoderDecoder for Statistical Machine Translation. arXiv preprint arXiv: 1406.1078.

Jeffrey Donahue, Lisa Anne Hendricks, Sergio Guadarrama, Marcus Rohrbach, Subhashini Venugopalan, Kate Saenko, and Trevor Darrell. 2015. Longterm Recurrent Convolutional Networks for Visual Recognition and Description. In CVPR, pages 2625-2634.

Akira Fukui, Dong Huk Park, Daylen Yang, Anna Rohrbach, Trevor Darrell, and Marcus Rohrbach. 2016. Multimodal Compact Bilinear Pooling for Visual Question Answering and Visual Grounding. EMNLP.

Yash Goyal, Tejas Khot, Douglas Summers-Stay, Dhruv Batra, and Devi Parikh. 2017. Making the V in VQA Matter: Elevating the Role of Image Understanding in Visual Question Answering. In $C V P R$, volume 1, page 9 .

Kaiming He, Xiangyu Zhang, Shaoqing Ren, and Jian Sun. 2015. Delving Deep into Rectifiers: Surpassing Human-Level Performance on Imagenet Classification. In ICCV, pages 1026-1034.

Kaiming He, Xiangyu Zhang, Shaoqing Ren, and Jian Sun. 2016. Deep Residual Learning for Image Recognition. In Proceedings of the IEEE conference on computer vision and pattern recognition, pages 770-778.

Sepp Hochreiter and Jürgen Schmidhuber. 1997. Long Short-Term Memory. Neural computation, 9(8):1735-1780.
Andrej Karpathy and Li Fei-Fei. 2015. Deep Visualsemantic Alignments for Generating Image Descriptions. In Proceedings of the IEEE conference on computer vision and pattern recognition, pages 3128-3137.

Diederik P Kingma and Jimmy Ba. 2015. Adam: A Method for Stochastic Optimization. In ICLR.

Ranjay Krishna, Yuke Zhu, Oliver Groth, Justin Johnson, Kenji Hata, Joshua Kravitz, Stephanie Chen, Yannis Kalantidis, Li-Jia Li, David A Shamma, et al. 2017. Visual Genome: Connecting Language and Vision Using Crowdsourced Dense Image Annotations. International Journal of Computer Vision, 123(1):32-73.

Qing Li, Jianlong Fu, Dongfei Yu, Tao Mei, and Jiebo Luo. 2018a. Tell-and-Answer: Towards Explainable Visual Question Answering using Attributes and Captions. arXiv preprint arXiv:1801.09041.

Qing Li, Qingyi Tao, Shafiq Joty, Jianfei Cai, and Jiebo Luo. 2018b. VQA-E: Explaining, Elaborating, and Enhancing Your Answers for Visual Questions. ECCV.

Xihui Liu, Hongsheng Li, Jing Shao, Dapeng Chen, and Xiaogang Wang. 2018. Show, Tell and Discriminate: Image Captioning by Self-retrieval with Partially Labeled Data. ECCV.

Jiasen Lu, Caiming Xiong, Devi Parikh, and Richard Socher. 2017. Knowing When to Look: Adaptive Attention via a Visual Sentinel for Image Captioning. In Proceedings of the IEEE Conference on Computer Vision and Pattern Recognition (CVPR), volume 6.

Pan Lu, Lei Ji, Wei Zhang, Nan Duan, Ming Zhou, and Jianyong Wang. 2018. R-vqa: learning visual relation facts with semantic attention for visual question answering. In Proceedings of the 24th ACM SIGKDD International Conference on Knowledge Discovery \& Data Mining, pages 1880-1889. ACM.

Ruotian Luo, Brian Price, Scott Cohen, and Gregory Shakhnarovich. 2018. Discriminability Objective for Training Descriptive Captions. In The IEEE Conference on Computer Vision and Pattern Recognition (CVPR).

Medhini Narasimhan, Svetlana Lazebnik, and Alexander Schwing. 2018. Out-of-The-Box: Reasoning with Graph Convolution Nets for Factual Visual Question Answering. In NIPS, pages 2655-2666.

Dong Huk Park, Lisa Anne Hendricks, Zeynep Akata, Anna Rohrbach, Bernt Schiele, Trevor Darrell, and Marcus Rohrbach. 2018. Multimodal Explanations: Justifying Decisions and Pointing to the Evidence. In $C V P R$.

Marco Pedersoli, Thomas Lucas, Cordelia Schmid, and Jakob Verbeek. 2017. Areas of Attention for Image Captioning. In ICCV-International Conference on Computer Vision. 
Jeffrey Pennington, Richard Socher, and Christopher Manning. 2014. Glove: Global Vectors for Word Representation. In Proceedings of the 2014 conference on empirical methods in natural language processing (EMNLP), pages 1532-1543.

Mengye Ren, Ryan Kiros, and Richard Zemel. 2015a. Exploring Models and Data for Image Question Answering. In NIPS, pages 2953-2961.

Shaoqing Ren, Kaiming He, Ross Girshick, and Jian Sun. 2015b. Faster R-CNN: Towards Real-time Object Detection with Region Proposal Networks. In NIPS, pages 91-99.

Steven J Rennie, Etienne Marcheret, Youssef Mroueh, Jarret Ross, and Vaibhava Goel. 2017. Self-critical Sequence Training for Image Captioning. In CVPR, volume 1, page 3 .

Yossi Rubner, Carlo Tomasi, and Leonidas J Guibas. 2000. The Earth Mover's Distance as a Metric for Image Retrieval. ICCV, 40(2):99-121.

Ramprasaath R Selvaraju, Michael Cogswell, Abhishek Das, Ramakrishna Vedantam, Devi Parikh, Dhruv Batra, et al. 2017. Grad-CAM: Visual Explanations from Deep Networks via Gradient-Based Localization. In ICCV, pages 618-626.

Damien Teney, Peter Anderson, Xiaodong He, and Anton van den Hengel. 2017. Tips and Tricks for Visual Question Answering: Learnings from the 2017 Challenge. arXiv preprint arXiv:1708.02711.

Ashwin K Vijayakumar, Michael Cogswell, Ramprasath R Selvaraju, Qing Sun, Stefan Lee, David Crandall, and Dhruv Batra. 2016. Diverse Beam Search: Decoding Diverse Solutions from Neural Sequence Models. arXiv preprint arXiv: 1610.02424 .

Oriol Vinyals, Alexander Toshev, Samy Bengio, and Dumitru Erhan. 2015. Show and Tell: A Neural Image Caption Generator. In Computer Vision and Pattern Recognition (CVPR), 2015 IEEE Conference on, pages 3156-3164. IEEE.

Jialin Wu, Dai Li, Yu Yang, Chandrajit Bajaj, and Xiangyang Ji. 2018. Dynamic Filtering with Large Sampling Field for Convnets. ECCV.

Jialin Wu and Raymond J Mooney. 2018. Faithful Multimodal Explanation for Visual Question Answering. arXiv preprint arXiv:1809.02805.

Jialin $\mathrm{Wu}$ and Raymond J Mooney. 2019. Selfcritical reasoning for robust visual question answering. arXiv preprint arXiv:1905.09998.

Jialin Wu, Gu Wang, Wukui Yang, and Xiangyang Ji. 2016. Action Recognition with Joint Attention on Multi-level Deep Features. arXiv preprint arXiv:1607.02556. 